\title{
Pinocchio, eroe di legno. Modelli mitologici,
} fiabeschi, realistici

Pinocchio, héros en bois. Modèles mythologiques, féeriques, réalistes

\section{Veronica Bonanni}

\section{OpenEdition}

\section{Journals}

\section{Edizione digitale}

URL: http://journals.openedition.org/cei/1040

DOI: $10.4000 /$ cei. 1040

ISSN: 2260-779X

\section{Editore}

UGA Éditions/Université Grenoble Alpes

\section{Edizione cartacea}

Data di pubblicazione: 31 octobre 2012

Paginazione: $229-240$

ISBN: 978-2-84310-234-9

ISSN: 1770-9571

Notizia bibliografica digitale

Veronica Bonanni, «Pinocchio, eroe di legno. Modelli mitologici, fiabeschi, realistici», Cahiers d'études italiennes [Online], 15 | 2012, online dal 30 avril 2014, consultato il 26 mars 2021. URL: http:// journals.openedition.org/cei/1040 ; DOI: https://doi.org/10.4000/cei.1040 


\title{
PINOCCHIO, EROE DI LEGNO. MODELLI MITOLOGICI, FIABESCHI, REALISTICI
}

\author{
Veronica Bonanni \\ Université de Lausanne
}

Spesso, come già notava Paul Hazard ${ }^{\mathrm{I}}$, i migliori libri per ragazzi sono approdati sullo scaffale delle librerie a loro riservati per «caduta» dallo scaffale degli adulti. Basti pensare a Robinson o a Gulliver, romanzi originariamente scritti per un pubblico adulto, ma dai quali i ragazzi si sono sentiti irresistibilmente attratti per il loro naturale spirito di ricerca e di avventura. Pinocchio, invece, ha seguito il percorso opposto: destinato ai "piccoli lettori» del Giornale per i bambini, è in seguito salito fino allo scaffale degli adulti, che se ne sono appropriati trasformandolo in un libro sapienziale denso di significati, in una trama inafferrabile e misteriosa capace di sottrarsi abilmente a ogni riduzione analitica ${ }^{2}$. A partire dal secolo scorso, infatti, la critica lo ha sottoposto alle interpretazioni più disparate - antropologiche, filosofiche, pedagogiche, psicologiche, e persino esoteriche, massoniche e teologiche — rivelando così l'inesauribile ricchezza e la straordinaria complessità di un'opera che il suo autore, certo incautamente, aveva definito una semplice «bambinata» ${ }^{3}$.

Il romanzo di Collodi, insomma, è ormai diventato un grande classico per tutti, un libro fruibile da un duplice pubblico di adulti e bambini, che possono leggerlo in maniera diversa a seconda dell'età. Ed è proprio tenendo conto di questa sua doppia destinazione che intendo accertare lo statuto di eroe (o antieroe) del protagonista, così come il suo valore di «modello» in una duplice accezione: modello da imitare (o evitare) per

I. P. Hazard, Les livres, les enfants et les hommes [1932], Paris, Éditions contemporaines Boivin \& C ${ }^{\mathrm{ie}}$, I949, p. 7I.

2. Come osserva Gianni Rodari nel suo intervento Pinocchio nella letteratura per l'infanzia, in Studi collodiani, Atti del I Convegno internazionale, Pescia, 5-7 ottobre 1974, Cassa di risparmio di Pistoia e Pescia, 1976, p. 40 .

3. Nel biglietto inviato a Biagi che accompagnava le prime cartelle della Storia di un burattino (I88I). 
i lettori, e modello letterario, di cui andranno rintracciati sia gli antecedenti, più o meno illustri, che gli eredi.

Per riflettere su queste due categorie, è bene partire dall'inizio, da quel celebre incipit in cui il narratore sembra prendersi gioco del suo lettore:

C'era una volta...

— Un re! — diranno subito i miei piccoli lettori.

- No, ragazzi, avete sbagliato. C'era una volta un pezzo di legno. ${ }^{4}$

Collodi comincia il suo racconto con un segnale inequivocabile di genere ("c'era una volta»), per poi rovesciarlo subito dopo: in questa fiaba moderna, a differenza dei vecchi contes de fées francesi che l'autore conosceva così benes, il protagonista non è un re, ma un pezzo di legno, per di più «un semplice pezzo da catasta, di quelli che d'inverno si mettono nelle stufe» ${ }^{6}$. Dove "pezzo da catasta" non indica solo il singolare aspetto fisico del personaggio, o certe sue qualità caratteriali — «legno da catasta», nella lingua parlata di allora, significava «birbante» ${ }^{7}$, ma anche la sua umile condizione sociale: Pinocchio, malgrado le sue sembianze, è un bambino povero, come ce n'erano tanti nell'Italia di quei tempi, che non potrà disporre di un vasto patrimonio come i suoi antenati principeschi, ma dovrà guadagnarsi il pane con le sue sole forze. Anche gli altri personaggi, del resto, si mostrano ben ancorati alla realtà sociale dell'epoca ${ }^{8}$, se solo si sfuma quell'alone di meraviglioso che li circonda: l'orco della fiaba diventa un marionettista, un mestierante del teatro, o un losco trafficante di bambini (l'omino di burro), e la fata, come già osservava Pancrazi ${ }^{9}$, si trasforma in una buona donnina del popolo, dopo essersi mostrata in un primo momento nel suo sfavillante apparato fiabesco. Ambientando il suo racconto in un luogo in cui è riconoscibile, anche se solo per qualche accenno, la Toscana di allora, popolata di contadini, mendicanti e truffatori, e dove ognuno si arrangia come può — si pensi al paese di Acchiappacitrulli, o al Gatto e alla Volpe - Collodi addomestica le fantasticherie senza tempo della fiaba per adeguarle alla società italiana contemporanea, con uno scivolamento del fiabesco verso il realismo del

4. C. Collodi, Le avventure di Pinocchio, in Opere, a cura di Daniela Marcheschi, Milano, Mondadori, I995, p. 36I (d'ora in poi il titolo sarà indicato con la sigla $P$., seguita dal numero del capitolo e della pagina).

5. Per averli tradotti in I racconti delle fate, Firenze, Paggi, I876, in cui figurano alcune fiabe di Perrault, D’Aulnoy e Leprince de Beaumont.

6 P., I, p. 36I.

7. Come suggerisce F. Tempesti, in C. Collodi, Pinocchio, introduzione e commento critico di F. Tempesti, Milano, Feltrinelli, I993, nota 2, p. I9.

8. Sul legame tra Pinocchio e la realtà sociale dell'epoca, si veda A. Asor Rosa, Collodi e De Amicis, in Storia d'Italia, Dall'Unità a oggi, Torino, Einaudi, I975, IV, 2, pp. 935-940.

9. P. Pancrazi, Venti uomini, un satiro e un burattino, Firenze, Vallecchi, I923, p. 201. 
romanzo. Tanto che il burattino di legno, circondato da adulti malintenzionati e costretto a lavorare duramente, pare quasi una versione nostrana dei bambini sfruttati e sfortunati messi in scena da Dickens.

Il rinnovamento della fiaba in Pinocchio, che fiaba in qualche modo resta ("c'era una volta»), ma aggiornata nei suoi temi, nei suoi personaggi, nel suo intreccio, e ibridata con i più svariati generi letterari — dal romanzo picaresco al racconto fantastico - comporta dunque innanzitutto un adeguamento del suo protagonista, che abbandona il lusso regale dell'oro per quel materiale assai più modesto che è il legno. $\mathrm{O}$, piuttosto, una preferenza per un certo tipo di personaggio, di bassa estrazione sociale, già presente anche nei contes de fées francesi dell'epoca di Louis XIV, ai quali Collodi in gran parte si ispira ${ }^{\mathrm{IO}}$. I modelli letterari, insomma, mi sembrano ancora in gran parte fiabeschi, ma riconducibili a un ben determinato tipo di fiaba. Gli antenati di Pinocchio non sono principi e principesse sfaccendati, ma bambini di un arcaico mondo rurale come quelli che si trovano in certi contes di Perrault. Il burattino rinvia così innanzitutto al Petit Poucet dell'omonimo racconto, figlio di due boscaioli "fort pauvres» " — così come poverissimo è Geppetto — che deve superare terribili prove, solo e abbandonato dai suoi genitori, e trovare un sostentamento economico per sé e per la propria famiglia; di lui, però, Pinocchio non possiede l'intuito e la fine intelligenza quasi adulta. La sua ingenuità, anzi, lo apparenta piuttosto al Petit Chaperon rouge, giovane campagnola sprovveduta e inesperta del mondo.

Quel «re» evocato e poi negato all'inizio del racconto, con cui si suscitano e poi si deludono le aspettative del lettore, non è quindi segno di una negazione del fiabesco, ma di un particolare tipo di fiabesco - quello permeato di magico e lontano dalla realtà sociale — che difficilmente poteva essere riproposto a scopo pedagogico. Ciò non significa, tuttavia, che il meraviglioso sia escluso dal romanzo di Collodi; significa, piuttosto, che è messo al servizio di un nuovo pubblico e di nuove esigenze educative.

I primi tre capitoli ${ }^{\mathrm{r}}$, infatti, sono quasi interamente dedicati alla nascita straordinaria di Pinocchio, fabbricato dalle abili mani di un falegname: uno spazio piuttosto ampio, nell'economia generale del racconto, già di

Io. $\mathrm{E}$ in molte altre fiabe, naturalmente: ma mi limito a quelle che Collodi conosceva sicuramente molto bene per averle tradotte.

II. Perrault, Le Petit Poucet, in Contes, édition critique de J.-P. Collinet, Paris, Gallimard, I98I, p. I9I.

I2. Si consideri, però, che nel manoscritto inviato da Collodi a Biagi per la pubblicazione sul Giornale per $i$ bambini il testo, corrispondente ai primi tre capitoli del libro, non presentava divisioni interne (v. O. Castellani Pollidori, Introduzione a Le avventure di Pinocchio, Pescia, Fondazione Nazionale C. Collodi, 1983, p. 22). 
per sé segno dell'importanza che l'origine riveste nella costruzione del personaggio.

La nascita del burattino, destinato a una vita misera e sventurata, rinvia a un modello letterario illustre: al demiurgo che crea un essere umano dando forma e vita a materia inerte ${ }^{13}$. Si pensi, ad esempio, agli uomini plasmati da Prometeo con acqua e terra ${ }^{\mathrm{I}}$, a Pandora forgiata da Efesto su ordine di Zeus ${ }^{15}$, o alla prima coppia umana creata da Dio nella Bibbia. Il primo demiurgo del romanzo, tuttavia, non si mostra all'altezza del suo compito: mastro Ciliegia vuole fare di quel pezzo di legno una gamba di tavolino, un semplice oggetto tra gli altri. E si meraviglia molto, col suo spirito pratico estraneo a fantasticherie fiabesche, quando, al momento di calare il primo colpo d'ascia, sente una vocina che gli dice: "Non mi picchiar tanto forte! » ${ }^{16} \mathrm{Al}$ primo «solennissimo colpo», la vocina di nuovo si lamenta: «Ohi! tu m'hai fatto male!» ${ }^{17}$; reazione simile, come ha notato Jacqueline Risset ${ }^{18}$, a quella di Polidoro nell'Eneide e di Pier delle Vigne nella Divina Commedia. Ciò che non si è sufficientemente sottolineato, però, è che questi episodi tragici sono riformulati da Collodi in chiave comica e parodica: a parlare non è più l'anima di un morto rivestita di legno, ma l'anima di un bambino che ha voglia di vivere e di divertirsi.

Neppure il secondo demiurgo, d'altra parte, si mostra molto più adatto al suo ruolo: Geppetto non vuole dar vita a un bambino, vuole semplicemente fabbricarsi «un burattino maraviglioso, che sappia ballare, tirare di scherma e fare i salti mortali ${ }^{19}$, col quale girare il mondo per guadagnarsi il pane. Non si tratta, certo, di un grande progetto di vita; ma è un progetto che attira il giovane pezzo di legno: danzare, giocare e far salti è tutto quello che un burattino, così come un bambino, può desiderare. Proprio per questo stavolta il pezzo di legno decide di collaborare, affidandosi remissivamente agli arnesi da falegname di Geppetto. Senza capire, tuttavia, che questo progetto, questo "contratto" di vita implica anche delle sue responsabilità nei confronti del padre, da assistere e mantenere nella sua vecchiaia.

13. Su questo argomento ho già fatto alcune osservazioni nel mio articolo Geppetto, demiurgo con la parrucca. Uno sguardo antropologico sulla nascita di Pinocchio, in "Hamelin. Note sull'immaginario collettivo", 6, gennaio 2003, pp. I5-I8.

I4. Cfr. Apollodoro, Biblioteca, I, 7, I e Ovidio, Metamorfosi, I, vv. 8I-82.

15. Cfr. Esiodo, Teogonia, vv. 570-584 e Opere, vv. 6I-82.

I6. P., I, p. 36I.

17. P., I, p. 362 .

I8. J. Risset, Pinocchio e Dante, in C'era una volta un pezzo di legno. La simbologia di Pinocchio. Atti del Convegno organizzato dalla Fondazione nazionale Carlo Collodi di Pescia, Milano, Emme edizioni, I98I, p. 96. I9. P., II, p. 364 . 
Non meno ridicolo di mastro Ciliegia, questo bizzarro vecchietto con la sua parrucca gialla, che sogna un futuro da artista girovago, non è dunque un vero demiurgo, come Prometeo, ma la sua versione comica e parodica. Versione che, probabilmente, passa attraverso l'abbassamento del mito operato nella tradizione fiabesca: già in Pinto Smauto di Basile, ad esempio, una ragazza - singolare caso di demiurgo al femminile — fabbrica un uomo impastandolo come una torta con mandorle e zucchero mescolate all'acqua di rose. Non si tratta, però, di un figlio, ma di un fidanzato, variante maschile (e comica) della statua di cui si innamora Pigmalione $^{20}$.

Lintento parodico, in Pinocchio, si rivela non solo nella scelta dei personaggi, ma anche nello sviluppo dell'azione. La creatura fabbricata da Geppetto si ribella al suo creatore come la prima coppia umana nella Bibbia; anzi, si ribella quando non è neppure del tutto creata, compiendo le sue prime monellerie: i suoi occhi, appena fatti, si muovono o si mettono a guardare con insistente fissità; il suo naso comincia a crescere come il ramo di un albero; la bocca si apre a ridere e a canzonare; le mani, una volta scolpite, afferrano con poco garbo la parrucca del babbo; e i piedi, dopo aver sferrato il primo calcio e aver fatto un po' di pratica nel camminare, lo portano dritto dritto fuori di casa.

La strabiliante precocità di Pinocchio, che mentre ancora sta nascendo si comporta come un bambino cresciuto, può essere letta come una nuova versione del vecchio topos, descritto da Curtius ${ }^{21}$, del puer senex, in cui un giovane possiede la saggezza di un vecchio, tanto che un bambino appena nato può parlare o agire come un adulto, rivelando così fin dalla nascita il suo destino di eroe. Una versione, però, chiaramente parodica, dal momento che il burattino, anziché dar prova di maturità, manifesta la sua indole disubbidiente, vispa e irrequieta — l'indole, insomma, dell'infanzia non ancora domata dall'educazione. Con un richiamo, assai probabile, al modello irriverente di Ermes, che quando è ancora in fasce ruba una parte delle mandrie di Admeto affidate ad Apollo ${ }^{22}$; anche se il furto della parrucca compiuto da Pinocchio, evidentemente, è impresa molto meno eroica.

La creazione demiurgica, la ribellione del figlio al padre, la frase pronunciata da Pinocchio morente appeso all’albero ( $\mathrm{Oh}$ babbo mioio! se

20. Cfr. G. Basile, Pinto Smauto (V, 3), in Lo cunto de li cunti, a cura di M. Rak, Milano, Garzanti, I986, p. 9I2: «avenno [Betta] sentuto dicere ca n'autra statua a li prieghe de no certo re de Cipro deventaie viva, tanto pregaie la dea d'ammore che la statua commenzaie ad aprire l'uocchie [...]».

2I. E. R. Curtius, Europäische Literatur und lateinisches Mittelalter, Bern, A. Francke Verlag, I948.

22. Cfr. Esiodo, Teogonia, v. 938; Apollodoro, Biblioteca, II, IO, 2 e Ovidio, Metamorfosi, 2, vv. 679-707. 
tu fossi qui...»), così come altri dettagli e personaggi del testo (la fataMadonna) sono secondo il cardinale Giacomo Biffi ${ }^{23}$ velate allusioni alla Bibbia, che tradirebbero un'ispirazione cristiana, anche se inconscia, del laico Collodi. A mio avviso, però, dei semplici riferimenti intertestuali non possono costituire la prova di un'intima adesione ideologica: se anche, infatti, non si può escludere la presenza dell'intertesto biblico, in un clima culturale permeato di cristianesimo, ciò che importa è stabilire su quale registro l'autore si è tenuto nel rielaborarlo. E il registro usato da Collodi, come ho appena fatto notare, è comico e parodico.

Geppetto, caricatura del demiurgo di ascendenza mitica e biblica, non riesce infatti a creare un essere umano compiuto - i figli dei demiurghi, di solito, nascono già adulti - o almeno un bambino in carne e ossa, in grado di ragionare e di apprendere dalla sua esperienza; ma soltanto un burattino, una "testa di legno» ${ }^{24}$ — come lo chiamerà il Grillo-parlante incapace di imparare dal suo vissuto e quindi di cambiare, di crescere. Per questa sua impossibilità di trasformarsi e di maturare, adattandosi alle regole sociali, Pinocchio è destinato alla sconfitta e alla morte.

$\mathrm{O}$, almeno, intrasformabile Pinocchio appare nella prima versione breve del romanzo, come già notava Emilio Garroni ${ }^{25}$. La Storia di un burattino pubblicata sul Giornale per $i$ bambini, in effetti, doveva originariamente terminare al capitolo Xv, con la morte del protagonista impiccato alla Quercia grande dagli «assassini», il Gatto e la Volpe. Solo più tardi, commosso dalle letterine dei "piccoli lettori», come vuole la leggenda, o più probabilmente vinto dalle insistenze di Biagi e Martini ${ }^{26}$, Collodi avrebbe deciso di proseguire il suo racconto per farlo evolvere verso una conclusione meno drammatica.

In quella prima stesura, la morte è quindi la conseguenza inevitabile, se non la giusta punizione, dell'atteggiamento irresponsabile e disobbediente del burattino. Tanto più che il sadismo pedagogico del finale, nel manoscritto di Collodi, era sottolineato da una moralité conclusiva, sul modello delle favole e dei conteurs francesi, soppressa nella rivista per volontà di Biagi e Martini: «Amici miei: avete dunque capito? Tenetevi lontani i cattivi compagni, e i libri cattivi: perché alla vostra età, un compagno cattivo o un libro cattivo possono esser molte volte cagione della vostra rovina.» ${ }^{27}$

23. G. Biffi, Contro mastro Ciliegia. Commento teologico a «Le avventure di Pinocchio», Milano, Jaca Book, 1977.

24. P., IV, p. 373 .

25. E. Garroni, Pinocchio uno e bino, Roma-Bari, Laterza, 1975.

26. Martini era il direttore del Giornale per i bambini, Biagi l'amministratore-factotum.

27. Cit. in P. Zanotto, Pinocchio nel mondo, Cinisello Balsamo, Edizioni Paoline, I990, p. 24. Il testo originale di Collodi è conservato al Museo Centrale del Risorgimento di Roma. 
Il primo Pinocchio, secondo il progetto dell'autore, era insomma un semplice racconto ammonitorio, un "conte de mise en garde» sull'esempio del Petit Chaperon rouge di Perrault, che, contrariamente ai Grimm, non dà una possibilità di salvezza alla vittima divorata dal lupo. Come Cappuccetto Rosso, Pinocchio muore per la sua ingenuità, che lo induce a fidarsi di sconosciuti malintenzionati. In quel breve racconto, che solo più tardi si sarebbe trasformato in romanzo, Collodi non voleva dunque proporre ai giovani lettori un modello da imitare, ma un antimodello, un esempio da evitare: se non si vuole fare la stessa fine di Pinocchio, suggerisce la lezione morale, non ci si deve comportare come lui.

Solo se si tiene conto di questo è possibile interpretare i primi capitoli in tutte le loro sfumature e comprenderne la funzione pedagogica: nelle scene iniziali si assiste infatti non alla nascita di un eroe, ma a quella di un antieroe, condannato alla miseria e alla morte. A rivelarlo, oltre al ribaltamento del mito demiurgico, è anche la rivisitazione di alcuni motivi fiabeschi: qui non c'è nessuna fata a presiedere alla nascita dell'eroe e a fargli doni; c'è solo Geppetto, che può donare al figlio unicamente un vestituccio di carta e scarpe fatte di scorza d'albero, simbolo, senz'altro, della «vocazione vegetale» di Pinocchio ${ }^{28}$, ma anche segno evidente di bassa condizione sociale. Pure il nome imposto da Geppetto, del resto, non è benaugurale, come invece lo sono tanti altri nomi altisonanti della tradizione fiabesca: «[...] lo voglio chiamar Pinocchio. Questo nome gli porterà fortuna. Ho conosciuto una famiglia intera di Pinocchi: Pinocchio il padre, Pinocchia la madre e Pinocchi i ragazzi, e tutti se la passavano bene. Il più ricco di loro chiedeva l'elemosina.» ${ }^{29}$

Il termine "pinocchio», suggerisce Fernando Tempesti ${ }^{30}$, significa in toscano "pinolo»: il nome del burattino, quindi, è un altro riferimento alla sua natura vegetale, diversa rispetto a quella degli esseri umani, che lo destina all'inadattabilità a questo mondo; ma è anche un'allusione al suo essere una piccola cosa, una cosa da nulla. Pinocchio, per il nome che porta, si rivela parente nostrano del Petit Poucet di Perrault, che tuttavia sarà capace, a differenza del burattino, di smentire le modeste aspettative suscitate da quel nomignolo poco promettente. E il lungo racconto della sua nascita, avvenuta nelle circostanze meno favorevoli, si può forse paragonare a quello di Tristram Shandy, antieroe nato sotto i peggiori auspici, il cui nome è una storpiatura del ben più altisonante Trismegistus.

28. Manganelli, Pinocchio: un libro parallelo [1977], Milano, Adelphi, 2002, p. 24.

29. P., III, p. 367.

30. F. Tempesti, Chi era il Collodi. Com’e fatto Pinocchio, in Pinocchio, Milano, Feltrinelli, I972, pp. IIO-III. 
Nella prima versione di Pinocchio in quindici capitoli, dunque, il destino di antieroe e la fine tragica del protagonista sono già scritti fin dalla sua nascita. Geppetto, demiurgo depotenziato e invecchiato, non riesce infatti a trasformare la natura vegetale e selvaggia dell'essere che ha creato. Anzi, non lo crea neppure, poiché il pezzo di legno è già vivo, come dimostra la sua «vocina» capace di spaventare mastro Ciliegia, prima ancora di assumere sembianze quasi umane ${ }^{31}$. Il falegname-demiurgo non dona a Pinocchio la vita, ma soltanto una nuova forma, che gli permette di muoversi più agevolmente ${ }^{32}$.

Il burattino che corre per le strade e per i campi, con le sue gambe legnose appena intagliate, conserva ancora tutta la sua vegetalità — rimando alla natura selvaggia, ribelle e anarchica dell'infanzia non ancora domata e «civilizzata» dagli adulti. è quindi un personaggio ben diverso rispetto ai bambini modello, obbedienti e studiosi, che si trovano nella letteratura per l'infanzia italiana dell'epoca. Ma quell'antimodello diverrà, a sua volta, un modello letterario per gli scrittori che si rivolgono ai ragazzi: basti pensare al Gian Burrasca di Vamba (pubblicato in volume nel I9I2) o alla Viperetta di Antonio Rubino (I919), due tra i più noti rappresentanti della stirpe monellesca di cui è capostipite il burattino.

Pinocchio è immagine dell'infanzia non solo per questa sua selvatichezza, simboleggiata da quel nasone che cresce a dismisura, ma anche per la sua maniera di pensare. Si può anzi affermare che, nonostante il suo aspetto burattinesco, è il primo, vero bambino della letteratura italiana, perché non pensa con la logica dell'adulto, ma con la logica dell'infanzia. Una logica difettosa, innanzitutto, nel riconoscere il principio di causalità: proprio perché non riesce a individuare le relazioni causali tra gli eventi, e di conseguenza la sua responsabilità nel determinarli, il burattino è persuaso di essere vittima innocente della malasorte, che gli fa piovere addosso continue disgrazie ${ }^{33}$.

Anche il senso del tempo e dello spazio appare diverso da quello degli adulti. Come tutti i bambini, Pinocchio si mostra incapace di andare al di là del momento presente, se non per progetti utopici che gli paiono di semplice e immediata realizzazione:

3I. Cfr. Manganelli, Pinocchio: un libro parallelo, cit., p. 30: "[Pinocchio] è nato nel momento in cui si staccava, erratico ramo, dalla sua pianta».

32. Non gli dona, però, la capacità di movimento: Pinocchio è capace di spostarsi, anche se piuttosto goffamente, pure quando è ancora un pezzo di legno (cfr. P., II, p. 365: «Mastr'Antonio, tutto contento, andò subito a prendere sul banco quel pezzo di legno che era stato cagione a lui di tante paure. Ma quando fu lì per consegnarlo all'amico, il pezzo di legno dètte uno scossone e sgusciandogli violentemente dalle mani, andò a battere con forza negli stinchi impresciuttiti del povero Geppetto.»)

33. Emilio Varrà, Albo e tempo, in Hamelin, Ad occhi aperti, Roma, Donzelli, 20I2, p. 94. 
Smesso che fu di nevicare, Pinocchio col suo bravo Abbecedario nuovo sotto il braccio, prese la strada che menava alla scuola: e strada facendo, fantasticava nel suo cervellino mille ragionamenti e mille castelli in aria, uno più bello dell'altro.

E discorrendo da sé solo diceva:

- Oggi, alla scuola, voglio subito imparare a leggere: domani poi imparerò a scrivere, e domani l'altro imparerò a fare i numeri. Poi colla mia abilità guadagnerò molti quattrini e coi primi quattrini che mi verranno in tasca, voglio subito fare al mio babbo una bella casacca di panno. Ma che dico di panno? Gliela voglio fare tutta d'argento e d'oro, e coi bottoni di brillanti. ${ }^{34}$

Il passato, invece, gli sembra qualcosa di molto vago, un unico insieme in cui non c'è molta differenza tra dieci e cent'anni e che non si riaggancia al presente in una continuità temporale, come mostra questo dialogo col Grillo:

- Io sono il Grillo-parlante, e abito in questa stanza da più di cent'anni.

- Oggi però questa stanza è mia — disse il burattino - e se vuoi farmi un vero piacere, vattene subito, senza nemmeno voltarti indietro. ${ }^{35}$

Il burattino non si stupisce per nulla della straordinaria longevità del Grillo, come se il passato non avesse profondità e durata, mentre limita il suo ragionamento al momento presente, all' «oggi», senza pensare che tale momento è legato al passato e ha necessarie ricadute sul futuro.

I luoghi da lui frequentati, inoltre, sarebbero difficilmente collocabili su una mappa; sono infatti luoghi simbolici - come il Paese dei Balocchi, o quello delle Api industriose - di cui non è tanto importante conoscere la posizione geografica, quanto il loro significato nel cammino pedagogico del protagonista. Certo, spazio e tempo indeterminati sono elementi tipici della fiaba; ma Collodi sa metterli al servizio del suo romanzo per ricostruire la logica dell'infanzia. Tanto che le birichinate e la disobbedienza di Pinocchio non paiono conseguenze del suo animo cattivo, ma della sua logica deficitaria.

L'autore è riuscito in questo modo a costruire un personaggio nel quale i piccoli lettori possono più facilmente identificarsi, rispetto ai bambini modello che ragionano come piccoli adulti. Ma ciò che più facilita l'immedesimazione, mi pare, è l'aspetto non umano, o non completamente umano, del protagonista. Come sostiene Leo Lionni, che nel suo albo illustrato Little blue and Little yellow ${ }^{36}$ ha raffigurato i due protagonisti

34. P., cap. IX, p. 386.

35. P., cap. IV, p. 37 I.

36. 1959 a New York, poi 1967 in Italia. 
come semplici macchie di colore, è «molto più facile per un bambino identificarsi nell'immagine di un topo, o di un pezzetto di carta colorata, piuttosto che in quella di un altro bambino", perché quel bambino può essere molto diverso da lui, ad esempio per il colore della pelle; mentre «se il protagonista del libro è un topo il problema non esiste, perché è un simbolo, come Piccolo Blu» ${ }^{37}$. È dunque proprio per il suo aspetto di burattino che Pinocchio è riuscito a diventare simbolo universale dell'infanzia, e che il romanzo di Collodi è stato tradotto pressoché in tutte le lingue: tutti i bambini, quale che sia il colore della loro pelle, possono identificarsi in un burattino.

Fino a questo momento, mi sono limitata ad analizzare le categorie di eroe e modello nella prima versione di Pinocchio in quindici capitoli. Ma queste categorie acquistano un ben diverso valore se si considera il romanzo nella sua interezza; se insomma, come direbbe Emilio Garroni, si passa da Pinocchio I a Pinocchio 2. Nel suo saggio Pinocchio uno e bino, Garroni distingue infatti tra quello che chiama Pinocchio $I-\mathrm{i}$ primi quindici capitoli, che inizialmente costituivano l'intero racconto - e Pinocchio 2, che non comprende soltanto il seguito, a partire dal capitolo xvi, bensì il romanzo per intero, fin dal primo capitolo. Questo perché il lettore, percependo il romanzo come un tutto unico, mette in relazione i primi capitoli con i capitoli seguenti, e finisce per dare ai primi un senso che dipende anche dai successivi. Per comprendere questo effetto di retroazione nel processo di lettura, basta pensare alla conclusione del capitolo Xv: il burattino che sembrava morto si rivela, nel girare pagina, ancora vivo; la lettura del capitolo Xvi si ripercuote dunque su quella del capitolo XV, che cambia completamente di senso.

Nel passare da Pinocchio I a Pinocchio 2, osserva Garroni, Collodi ha dovuto modificare la natura del protagonista, facendolo diventare, da immutabile qual era, capace di trasformarsi e di imparare. Senza questo fondamentale adattamento, infatti, Pinocchio sarebbe stato comunque destinato, dopo aver vissuto altre picaresche avventure, allo stesso finale tragico del capitolo Xv. I suoi cambiamenti sono rappresentati come nascite e rinascite successive, che si possono considerare dei veri e propri «riti di passaggio" ${ }^{38}$, secondo la definizione di Van Gennep. Non si tratta però sempre di metamorfosi concrete, per cui il pezzo di legno si ritrova burattino, poi asino, poi bambino in carne ed ossa; Pinocchio rinasce simbolicamente uscendo dal ventre della balena, e diviene altro da sé anche

37. Cit. in A. Rauch, Tracce per una storia dell'albo, in Hamelin, Ad occhi aperti, cit., p. 6.

38. A. Van Gennep, Les rites de passage, Paris, Nourry, 1909. 
semplicemente assumendo un diverso ruolo (quando, ad esempio, prende il posto del cane Melampo), apparendo sotto un altro aspetto agli occhi degli altri (il Pescatore Verde lo scambia per un granchio) o anche soltanto nelle loro intenzioni (quando il suo compratore vuole farne una pelle di tamburo) ${ }^{39}$.

$\mathrm{Ma}$, se il rito di iniziazione ha certamente ascendenze fiabesche, la struttura generale del romanzo, in cui il protagonista, dopo numerosi errori commessi nella sua giovinezza, riesce a superare la propria ingenuità e ad adattarsi alle regole sociali, si rifa invece al romanzo di formazione europeo, di cui si trovano tracce anche nell'evoluzione del personaggio di Renzo nei Promessi sposi $i^{4}$. Mentre, quindi, la prima versione breve di Pinocchio era un semplice racconto ammonitorio, senza alcun vero percorso educativo, la seconda versione lunga diventa un romanzo di formazione, che annulla o ingloba gli effetti della prima, dal momento che le punizioni hanno effetti solo transitori. Così Pinocchio, che nel racconto originario era un antieroe, un antimodello, può ora diventare un eroe, un modello da seguire; non nel senso che il lettore deve imitare le sue azioni e i suoi comportamenti, ma nel senso che deve intraprendere il suo stesso cammino per imparare, come lui, a correggere i propri errori.

In questo modo il romanzo di formazione, che nella tradizione europea è essenzialmente realista, diventa qui un romanzo fiabesco, in cui il meraviglioso si fonde con il realismo. Se, infatti, si prescinde dalla sua veste meravigliosa, Pinocchio appare come la storia di un bambino vivace ed egoista che diventa un ragazzo responsabile, capace di aiutare gli altri, e che grazie al suo duro lavoro riesce a cambiare la propria condizione sociale, trasformandosi da poveraccio in piccolo borghese. Le avventure bel burattino costituiscono così un romanzo di formazione "anticipato", secondo la definizione di Milena Bernardi, in cui l'apprendistato non si colloca più nell'età giovanile, ma in quella infantile ${ }^{4 \mathrm{I}}$.

Anche se, a dire il vero, il percorso formativo di Pinocchio non sembra mantenersi strettamente nei limiti dell'infanzia. Il burattino che, rinato dal ventre della balena, deve accudire suo padre, portandolo in giro in una specie di carrozzina, e mantenerlo lavorando a ritmi forzati, diventa a sua volta padre, compiendo così con un ritmo accelerato il percorso di vita di ciascun uomo. Proprio per questo anche i lettori adulti possono

39. Sulle continue metamorfosi di Pinocchio insiste Manganelli (Pinocchio: un libro parallelo, cit.).

40. Per un paragone tra il personaggio di Collodi e di Manzoni, si veda E. N. Girardi, Pinocchio come Renzo, in Studi collodiani, cit., pp. 315-22.

4I. M. Bernardi, Il cassetto segreto. Letteratura per l'infanzia e romanzo di formazione, Milano, Unicopli, 201. 
identificarsi nel protagonista, che assume davvero un valore universale: il burattino non è solo immagine dell'infanzia, ma immagine dell'uomo. Si spiega così, dunque, come mai Pinocchio abbia conquistato anche il pubblico degli adulti, al quale non è esplicitamente diretto.

Pinocchio, insomma, si può leggere in due modi diversi: come la storia di un burattino che diventa un bambino perbene, o come la storia dell'uomo, obbligato a rinunciare alla sua libertà primitiva per sopravvivere in questo mondo. La nascita demiurgica del suo protagonista, d'altra parte, se riletta e riconfigurata alla luce dell'intera struttura del romanzo, non fa che avvalorare questa interpretazione: il destino di Pinocchio è il destino del primo uomo, così come di tutta l'umanità. 